MKG-Chirurg 2021 • 14:255-257

https://doi.org/10.1007/s12285-021-00311-8

Angenommen: 30. Juni 2021

Online publiziert: 24. August 2021

๑) Springer Medizin Verlag GmbH, ein Teil von

Springer Nature 2021

\section{Laudatio für Herrn Prof. Dr. Dr. Rudolf Reich anlässlich der Verleihung der Ehrenmitgliedschaft der DGMKG}

Hans-Peter Ulrich

MKG-Chirurgie LindenArcaden MVZ GmbH, Lübeck, Deutschland
Die Deutsche Gesellschaft für Mund-, Kiefer- und Gesichtschirurgie (DGMKG) verleiht satzungsgemäß und nach Vorstandsbeschluss die Ehrenmitgliedschaft an Herrn Prof. Dr. Dr. Rudolf Reich. Dies wurde im vergangenen Jahr beschlossen und coronabedingt auf der diesjährigen Mitgliederversammlung vollzogen (• Abb. 1).

Die beruflichen Stationen Herrn Prof. Reichs seien hier kurz rekapituliert. Nach zahnärztlicher und ärztlicher Approbation in Mainz und doppelter Promotion eben dort schloss er die Facharztausbildung 1983 in Hannover ab, daraufhin erhielt er die Gelegenheit zu einem von der Deutschen Forschungsgemeinschaft geförderten Studienaufenthalt in San Antonio, Texas, USA. Es folgte die Ernennung zum Leitenden Oberarzt, die Habilitation 1987, die Verleihung des Waßmund-Preises (heute Wissenschaftspreis der DGMKG) 1988, die C2- und außerplanmäßige Professur an der Medizinischen Hochschule Hannover und schließlich 1993 die Berufung auf den Lehrstuhl und die Ernennung zum Direktor der Poliklinik und Klinik für Mund-, Kiefer- und Plastische Gesichtschirurgie der Universitätsklinik Bonn.

Seit seiner Emeritierung 2018 ist es nicht ruhiger geworden, weil er die Aufgaben im Kopf-Hals-Tumor-Zentrum am Waldkrankenhaus Bonn, in der speziellen Kiefergelenksprechstunde und -chirurgie an der St. Lukas Klinik Solingen, im Medizinischen Versorgungszentrum Rheinzahn in Bonn und als Gutachter für die Ärztekammer Nordrhein in der Kommission für ärztliche Behandlungsfehler (der Schlichtungsstelle) und als Obergutachter der Kas- senärztlichen Bundesvereinigung für Ausnahmeindikationen der Implantologie gemäß § 28 SGB V mit viel Einsatz und Erfolg wahrnimmt.

Beispiele seiner exponierten wissenschaftlichen Tätigkeit sind:

- 1993 Gründungsmitglied der Deutschen Gesellschaft für Schädelbasischirurgie

- 2009 Gründungsmitglied der European Society of Temporomandibular Joint Surgeons

- 2014-2016 Präsident der European Society of Temporomandibular Joint Surgeons, 2015 deren Jahreskongresspräsident in Bonn

Für die DGMKG hat Herr Prof. Reich Hervorragendes geleistet. Die Aufzählung all dessen würde den Rahmen dieser Laudatio bei Weitem sprengen. Vielmehr soll mit wenigen herausgehobenen Punkten schlaglichtartig Herrn Prof. Reichs Wirken skizziert werden.

1989 wurde er auf Vorschlag Herrn Prof. Scheunemanns zum Generalsekretär der DGMKG gewählt - die Bitte, damals wahrscheinlich eher ein Auftrag, erfolgte am Tag zuvor. Diese Funktion hat Herr Prof. Reich in hervorragender Weise ausgefüllt. Sein Lehrer, Herr Prof. Hausamen, war der aktuelle Präsident der DGMKG. Konsequent war damit auch Herrn Prof. Reichs Funktion im Vorstand der DGMKG verbunden.

Die wohl wichtigste Entwicklung der DGMKG war die Vorbereitung und erfolgreiche Umsetzung der Fusion mit dem Berufsverband. Hier hat Herr Prof. Reich entscheidend mitgewirkt, wohl wissend, dass dies nur unter Hintanstellung persönlicher 


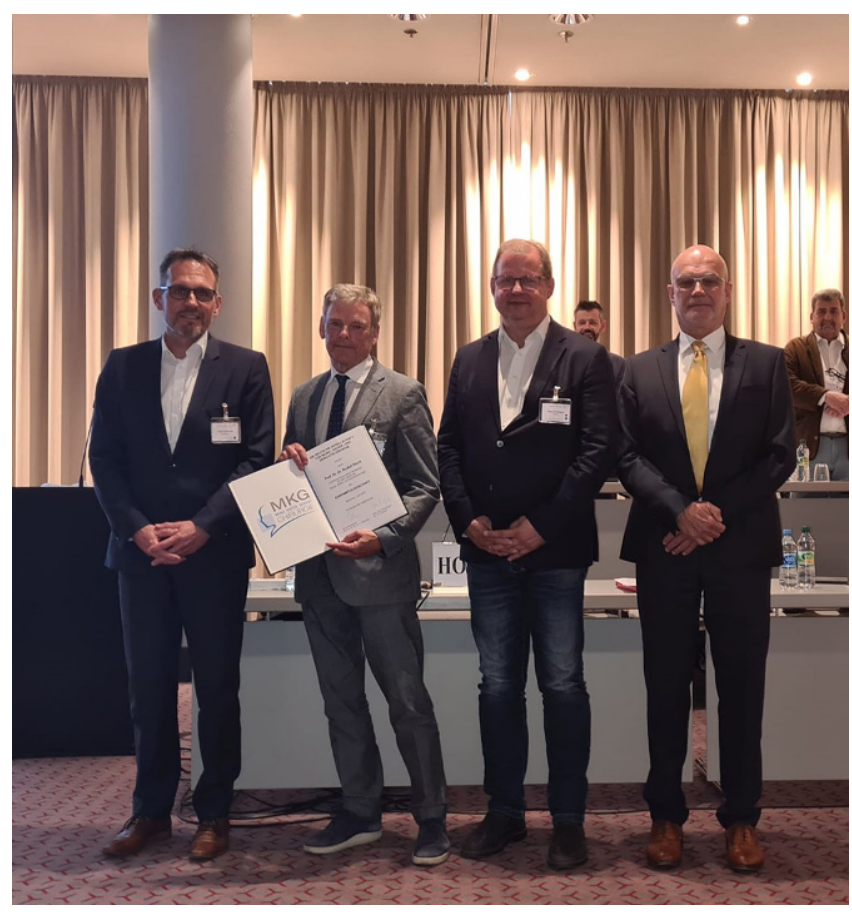

Abb. $1 \varangle$ Verleihung der Ehrenmitgliedschaft an Herrn Prof.Dr.Dr.Rudolf Reich (v. I. n. r.: Prof. Dr. Dr. Dr. h.c. Jürgen Hoffmann, Präsident DGMKG, Prof.Dr. Dr. Rudolf Reich, Ehrenmitglied DGMKG, Dr. Jörg Wiegner, Vizepräsident DGMKG, Dr. Dr. Hans-Peter Ulrich, Laudator)

Interessen möglich war. Er wäre der vorgesehene Präsident im Jahr 2000 gewesen, wurde jedoch erst nach der Fusion für die Jahre 2004-2006 in das Amt gewählt. Dass er als Vizepräsident von 2002 bis 2004 mitgetragen hat, auch gegen Widerstände, dass ein Nichthabilitierter und im ambulanten Bereich Tätiger erstmals Präsident der DGMKG wurde, war nicht selbstverständlich.

Die DGMKG hat in wesentlichen Teilen Herrn Prof. Reich zu verdanken, dass die Fusion der Gesellschaft erfolgreich war und ist. Einige der vielen Leistungen und Maßnahmen, an denen Herr Prof. Reich entscheidend beteiligt war:

- Abstimmung des ersten Katalogs ambulanter Operationen

- Stellung der MKG-Chirurgie im Bundeswehrsanitätsdienst

- Optimierung des Auswahlverfahrens für den Waßmund-Preis (heute Wissenschaftspreis der DGMKG)

- Optimierung der Auswahlverfahren und Organisation der Vorträge am Jahreskongress, dessen Ablauf und Verträge inkl. Wehr- und Katastrophenmedizinsymposium

- Begleitung der Selbstständigkeit des Deutsch-Österreichisch-Schweizerischen Arbeitskreises für Tumoren im Kiefer- und Gesichtsbereich 2005
- Verankerung der MKG-Chirurgie in zertifizierten überregionalen Traumazentren der Deutschen Gesellschaft für Unfallchirurgie und damit Übernahme in das Weißbuch der Schwerverletztenversorgung, dadurch Festigung der stationären MKG-Chirurgie in diesen Kliniken der Maximalversorgung

- Für die DGMKG wahrgenommene Funktionen als Delegierter bei der Akademie der Fachärzte und der Arbeitsgemeinschaft der Wissenschaftlichen Medizinischen Fachgesellschaften sowie Vertreter in der AHMO (Arbeitsgemeinschaft Hals-Nasen-OhrenHeilkunde, Mund-Kiefer-Gesichtschirurgische Onkologie)

Hervorzuheben ist, dass Herr Prof. Reich, neben der Organisation des Jahreskongresses 2017 in Bonn, gemeinsam mit Herrn Prof. Zöller, auch entscheidend die Europadelegation unserer Gesellschaft mitgeprägt hat. Als Präsident der Educational Commission des European Board of Oro-Maxillo-Facial Surgery (EBOMFS) hat er dazu beigetragen, für die deutsche MKG-Chirurgie abträgliche Vorstellungen einzugrenzen und angelsächsisch geprägte Regularien im Wesentlichen zu verhindern. Die Teilnehmer der EBOMFSAssessments in Dubrovnik 2012, Prag 2014, London 2016 und München 2018 haben sehr davon profitiert. Den dafür erforderlichen immensen Aufwand können nur Insider erahnen. Nicht zuletzt hat er an der Interpretation des Wording Annex V PQD (Professional Qualifications Directive EU 2005/36 ff.) mitgewirkt, sodass unsere Interpretation vom Gesundheitsministerium übernommen wurde:

Das Zahnmedizinexamen muss erst zum Abschluss der Facharztweiterbildung vorliegen, was die Weiterbildungszeit erheblich verkürzen kann.

Ein paar Worte seien zum Persönlichen erlaubt. Herr Prof. Reich hat einen der Nestoren unserer Gesellschaft, Herrn Prof. Scheunemann, gleich zu Beginn seines Studiums kennengelernt und wurde von ihm gefördert. Diese Verbundenheit hat sich bis in die letzten Stunden Herrn Prof. Scheunemanns erhalten und ist Ausdruck einer gegenseitigen Wertschätzung, wie sie heute selten zu finden ist. Herr Prof. Hausamen, der auch Ehrenmitglied der DGMKG ist, hat Herrn Prof. Reich sehr geprägt. Die Verleihung der Ehrenmitgliedschaft an Herrn Prof. Rudolf Reich steht in dieser Tradition. Vermittelt wurde konfuzianische Haltung: „Fordere viel von dir selbst und erwarte wenig von anderen."

Herr Prof. Reich hat sich immer für die fusionierte DGMKG eingesetzt, auch gegen den Widerstand und die Vorstellung einiger Kollegen. Die mehr als verdiente Verleihung der Ehrenmitgliedschaft an Herrn Prof. Reich ist eine Würdigung seiner Leistung und seines Verhaltens.

Seine Leistungen sind nur mit der Unterstützung im privaten Umfeld möglich geworden. Die beiden Kinder Julius und Stella sind inzwischen beruflich erfolgreich und haben das Engagement des Vaters offensichtlich unbeschadet getragen und überstanden. SeineFrau Sibyllehat ihn, wie er selbst sagt, mehr als 31 Jahre immer geerdet und unterstützt. Die inzwischen betagte Border-Collie-Dame Paula trug ihren Anteil zum Ausgleich zwischen Arbeit und Familie bei, was Picasso perfekt beschrieb: "Hunde kommen in unser Leben, um zu bleiben. Sie gehen nicht fort, wenn es schwierig wird, und auch, wenn der erste Rausch verflogen ist, sehen sie uns noch immer mit genau diesem Ausdruck in den Augen an." 
Lieber Rudolf, wenn jemand verdient hat, Ehrenmitglied zu werden, dann Du.

Glückwunsch!

Hans-Peter Ulrich

\section{Korrespondenzadresse}

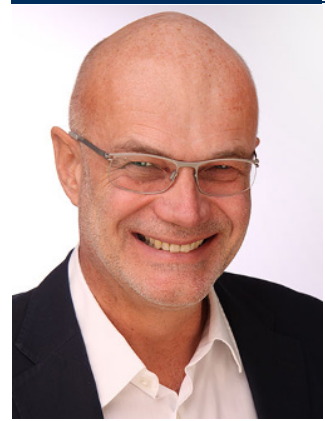

Dr. Dr. Hans-Peter Ulrich

MKG-Chirurgie LindenArcaden MVZ GmbH

Fackenburger Allee 1, 23552 Lübeck,

Deutschland

ulrich@mkg-lindenarcaden.de
Hier steht eine Anzeige.

Springer 